\title{
An IP-Level Analysis of Usage Statistics for Electronic Journals in Chemistry: Making Inferences About User Behavior
}

\author{
Philip M. Davis \\ Albert R. Mann Library, Cornell University, Ithaca, NY 14853. E-mail: pmd8@cornell.edu \\ Leah R. Solla \\ Physical Sciences Library, Cornell University, Ithaca, NY 14853. E-mail: Irm1@cornell.edu
}

\begin{abstract}
This study reports an analysis of American Chemical Society electronic journal downloads at Cornell University by individual IP addresses. While the majority of users (IPs) limited themselves to a small number of both journals and article downloads, a small minority of heavy users had a large effect on total journal downloads. There was a very strong relationship between the number of article downloads and the number of users, implying that a user-population can be estimated by just knowing the total use of a journal. Aggregate users (i.e. Library Proxy Server and public library computers) can be regarded as a sample of the entire user population. Analysis of article downloads by format (PDF versus HTML) suggests that individuals are using the system like a networked photocopier, for the purposes of creating print-on-demand copies of articles.
\end{abstract}

\section{Introduction}

Both publishers and librarians are rapidly moving into evaluating the worth and utility of their journal subscriptions with usage statistics. Without understanding online use behavior, any quantitative evaluation can be easily misinterpreted. Until we can better understand the intent of the user and be able to represent it numerically, most attempts to derive value and utility from use statistics will be greatly compromised.

Most of what we currently know about user behavior of journal literature comes from citation analysis and library circulation studies. Both of these methods attempt to estimate journal use, but are problematic in different ways. Citation analysis does not measure use directly, but estimates it through the citation patterns of authors. Library

Received February 3, 2003; revised March 17, 2003; accepted March 17, 2003

() 2003 Wiley Periodicals, Inc. circulation studies are a more direct form of measuring use but are prone to underestimate journal use, greatly underestimate article use, and ignore the use of personal subscriptions.

Survey research is also a useful tool in understanding user behavior, yet the construction of surveys is partly subjective. And while surveys generate quantifiable data, they rely on the subject to accurately and honestly report his/her own practices. In-depth interviews-while qualitative in nature - can garner details, motivations and beliefs, and provide us with a better understanding of the rationale behind the behavior itself. Because of time and expense, interviews are limited in scale, and also may be prone to bias by both the interviewer and interviewee.

Usage statistics more directly measure use than citation analysis; however, most publisher and vendor reports:

- do not tell us what is being downloaded;

- do not tell us why an article was downloaded;

- do not tell us how many are responsible for the statistics; and

- cannot account for use of personal or print subscriptions.

Adding to the problem of interpretation, publishers currently count and report usage statistics in many different ways. This becomes very problematic when a librarian needs to analyze the use of journal titles in a particular subject discipline, compare the use of databases, or conduct any systematic collection review. Project COUNTER, an international group of publishers and librarians, was created with the goal to develop an "internationally accepted, extendible Code of Practice ... that will enable vendors to provide these requested statistics in a way which all parties can trust to be consistent, credible and compatible." The first Code of Practice was released in December, 2002 (COUNTER, 2003).

To date, most online publishers have only provided total usage statistics for journal titles, either summarized 
monthly, quarterly, or annually. Consequently, most published studies have focused on aggregated data analysis. Very little is known about usage patterns of individuals and how they contribute to these aggregated statistics.

\section{Problem with Identifying and Measuring Individual Users}

Although the concept of individual user is not difficult to comprehend, it is extremely difficult to measure in the current online environment. Librarians, on principle, have defended their patrons' right to confidentiality and have successfully argued against required logins that would enable researchers to directly measure individual behavior.

The goal of this paper-to better understand the nature of individual online behavior-cannot be achieved by directly measuring individual use. Because of this limitation, the authors will approximate individual use by utilizing individual IP addresses as a surrogate measure. While an IP address denotes an individual computer and not necessarily an individual user, it will be used to gain a better understanding of individual online behavior beyond what is currently known.

This study reports an IP analysis of twenty-nine online scholarly journals from the American Chemical Society (ACS). Although limited to a single, specialized publisher, it is believed that the patterns of individual use described in this article may be generalizable to other scientific journals. The ultimate utility of this study is to be able to apply these findings to other publisher data sets where only aggregate use data are available.

\section{Literature Review}

To date, relatively few articles have focused on the analysis of e-journal data. Tom Sanville has focused his analysis at the level of the OhioLink Consortium, aggregating statistics for each institution or for each publisher (Sanville, 2001). The author recently reported a study on the use-patterns exhibited between institutions for single publisher, and focused on the journal as the subject of analysis (Davis, 2002). Carol Tenopir and Deborah Blecic have both reported on temporal variation of database and journal use for institutions, indicating peak use by day, month, and cumulative change over time (Blecic, Fiscella, \& Wiberly, Jr., 2001; Tenopir \& Read, 2000). David Nicholas and others recently reported a log-file analysis of the UK publisher, Emerald, reporting aggregated statistics such as percentages of downloads from subscribing institutions, format preferences, and time to download articles (Nicholas, Huntington, \& Watkinson, 2002). To date, the most thorough study to date that explores the online journal use of individuals comes from the SuperJournal project.

\section{Experiences from SuperJournal}

The SuperJournal project was an experiment in the late 1990 s to better-understand user-behavior of electronic jour- nals (Pullinger, 1994). SuperJournal was a web-based system consisting of forty-nine scholarly journals from nine publishers and delivered to thirteen participating sites in the UK. Individuals using SuperJournal were required to register and provide basic demographic information about themselves, allowing individual behavior to be tracked. Post hoc interviews and questionnaires were distributed to gather qualitative information on user experience (Eason, Richardson, \& Yu, 2000).

Based on analysis from their log files, users were classified according to their frequency of use, breadth of use, and depth of use. Their analysis identified four repeat user groups: the enthusiastic user, the focused regular user; the specialized occasional user, and the restricted user; and three groups of non-repeat users: the lost user; the exploratory user; and the tourist.

Post-hoc interview and web questionnaire surveys illustrated that when relevant articles were found; the majority of users would print them. Only a small minority of users would read the article on screen. It was also found that users prefer HTML format when they read on the screen and PDF format when they print. The authors speculated that a comparison of the two might provide an indication of the browsing versus printing behavior of patrons.

\section{Understanding the Reading Behavior of Scientists}

Usage statistics are only meaningful when understood within the context of user behavior. Both the time spent reading and the number of articles read varies considerably across subject disciplines. In general, chemists are well known to be very heavy users of the journal literature. Based on early citation studies of chemical source journals, $93.6 \%$ of citations were to journals, compared to $90.8 \%$ in physiology, $88.8 \%$ in physics, and $76.8 \%$ in mathematics (C.H. Brown, 1956).

Studies from 1977 through 2001 by Carol Tenopir and Donald W. King indicate that scientists currently read approximately 130 articles per year, with medical researchers reading the most articles and engineers reading the least (Tenopir \& King, 2002). Based on their surveys, chemists read an average of 276 articles per year, physicists an average of 204 articles per year, and engineers an average of 72. Medical researchers reported reading an average of 322 articles per year, yet spent only about 20 minutes per article, so that their total time reading is considerably less than chemists. Chemists reported spending more time reading (198 hours/year) than medical researchers (118), Physicists (153) or Engineers (92).

Scientists read some journals extensively, and others very rarely. For high-use journals, scientists are more likely to use personal subscriptions over library subscriptions (Tenopir \& King, 2000). A 1998 study of physical scientists reported that over half of chemists $(55 \%)$ used personal subscriptions to obtain journal articles, compared to $23 \%$ for mathematicians, and $38 \%$ for physicists and astronomers (C.M. Brown, 1999). Therefore, the effect of personal sub- 
scriptions when measuring institutional user statistics may be problematic, having the effect of under-representing the use of popular browsing journals.

In the early 1990s, Jan Olsen conducted in-depth interviews of chemists, sociologists and English faculty at Cornell University and the University of Pennsylvania (Olsen, 1994). Olsen's study illustrated that chemists employ techniques to deal with a great deal of published literature. Chemists indicated that they usually read the parts of an article out of order, focusing on the abstract, figures, and captions. Chemists also indicated that they rarely read an entire article unless it was extremely relevant to their work (Olsen, 1994).

CORE (Chemistry Online Retrieval Experiment) was an online experiment in providing full-text and full-image of twenty ACS journals (Entlich et al., 1995). Based on interviews of 39 CORE users at Cornell University, the most important feature of the system was the ability to make a printed copy ( $80 \%$ considered it very important), followed by the ability to browse graphics (73\%), and ability to browse text (66\%) (Stewart, 1996).

For a thorough description of the research on the reading behavior of scholars, consult the review article by King and Tenopir (1999).

\section{Dataset}

The dataset under investigation is composed of electronic journal use statistics for 29 scholarly journals from the American Chemical Society, at Cornell University. A list of ACS journals can be found from their Web site. The dataset covers a three-month window July-September 2002 (see Representative Time Frame below). The statistic observed in this study is a full-text article download (either in HTML or PDF format). Use-statistics for each journal were summarized for each unique IP address. The dataset does not include online use by personal subscriptions.

\section{Definitions and Assumptions}

IP address. An IP (Internet Protocol) address is a numeric identification given to each networked computer. It is assumed for simplicity and for purposes of this study that a single IP address represents a single user, with some exceptions (see User). Individuals who dial-in to the Cornell network using a modem, or use the wireless network are assigned a temporary IP address.

User. In this study, the term "user" will used to describe the behavior of an individual IP address. In order to maintain patron confidentiality, no attempt was made to identify the owner of any computer, although department affiliation was identified. Some computers are shared, such as those in public labs or libraries. The Library Proxy Server is a computer that facilitates access to Cornell-restricted electronic resources when patrons connect from outside the campus network and thus represents an aggregate of individual users. Cornell University also maintains a modem pool for individuals dialing into the campus network by telephone.

Representative time frame. The dataset represents Cornell University use-statistics for the third quarter, July-September, 2002. While our use-statistics for ACS journals have been slowly increasing over the last year and a half, the proportion of use across journals has remained relatively constant. Total journal downloads for the third quarter correlated extremely highly with first and second quarters ( $R$ $=0.984$ after a log transformation of raw use). Therefore, despite the fact that this dataset covers the summer months, it should be considered a good representative sample of other time periods for Cornell.

Inferences on user behavior. Unlike other studies that rely on questionnaires, interviews or transactional log analysis, we are unable to directly ascertain the purpose or sequence of events that generated the use statistics analyzed in this article. Any statement-beyond quantitative-we make on user behavior is therefore inferred and not directly observable.

Outliers. Outliers are defined as observations that differ greatly from expected results, and have a great deal of effect or leverage on a statistical model. In real terms, they either represent observational error, or some unusual behavior that needs to be explained. Outliers can have a large influence on total or aggregated use-statistics. For example, if most users download one or two articles during the observation, a single individual who downloads hundreds of articles from a single journal would be considered an outlier and may need to be explained as unusual behavior.

\section{Observations}

In the dataset, we identified 1,283 individual IP addresses responsible for downloading a total of 23,863 articles from 29 ACS journals.

\section{Aggregate Use}

IP addresses associated with the departments of Chemistry and Chemical Engineering were responsible for $42 \%$ of the downloads, followed by Engineering (12.5\%, Civil, Biological and Environmental, Materials, and Theoretical), Food Science (4.9\%) and Molecular Biology \& Genetics $(2.6 \%)$. The Medical College campus_physically separated from the main college campus-accounted for $6.5 \%$ of article downloads. The Library Proxy Server, an aggregate "user," was responsible for 1,032 (4.3\%) article downloads. Taken together, articles downloaded from computers in libraries totaled $805(3.4 \%)$. Individuals dialing into the 


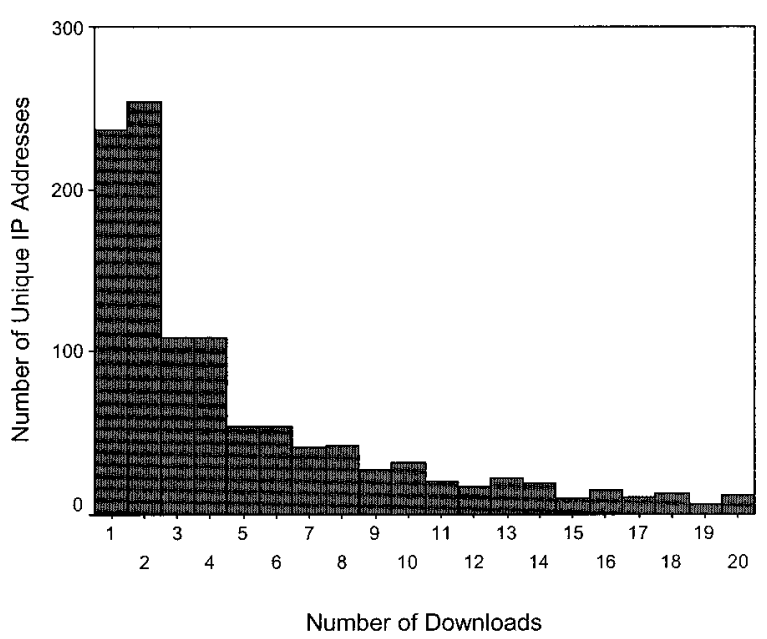

FIG. 1. Number of article downloads by IP $(\mathrm{N}=1,283)$.

Cornell's network modem pool, were only responsible for $355(1.5 \%)$ article downloads.

\section{Individual Use: Articles}

A histogram illustrating the frequency of downloads by IP (Fig. 1) shows a rapidly decaying distribution. Thirtyeight percent $(38 \%)$ of all users downloaded 1 or 2 articles during the three-month window; $16.8 \%$ of users downloaded 3 or 4 articles; $8.4 \%$ of users downloaded 5 or 6 articles; $14 \%$ of users downloaded more than 20 articles; and $3 \%$ of users downloaded more than 100 articles.

As expected, users in certain departments were associated with downloading more articles than others. Eightytwo percent $(82 \%)$ of IPs originating from the departments of Chemistry and Chemical Engineering downloaded more than 2 articles during the observed period, compared to $79 \%$ for Engineering, 69\% for Food Science, 53\% for the Medical College and $50 \%$ for Molecular Biology \& Genetics. Users dialing in to the Cornell campus using a modem connection downloaded very few articles in general. Only $43 \%$ of modem-users were associated with downloading more than two articles. Since modem pool IP addresses are shared among multiple dial-in users, these figures should be considered inflated.

\section{Individual Use: Journals}

Most users concentrated their use on a small number of titles (Fig. 2). Fifty-five and a half percent $(55.5 \%)$ of all users downloaded articles from just one journal; $18.7 \%$ of users downloaded from 2 journals; $8.9 \%$ of users downloaded from 3 journals; and just $1.9 \%$ of users downloaded articles from 10 or more journals. The Library Proxy Server used 24 of the 29 ACS journals.

Also as expected, certain departments were associated with consulting more journals than others. Sixty-six percent

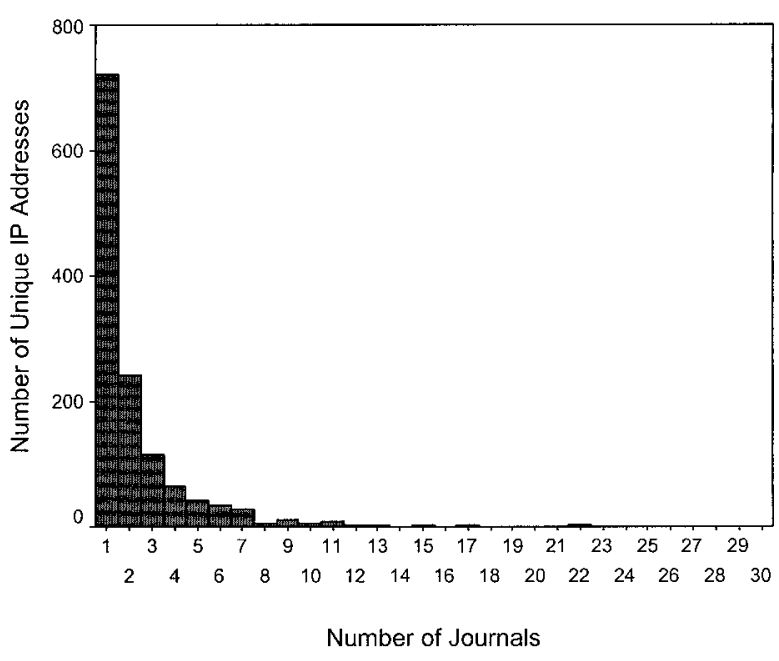

FIG. 2. Number of journals consulted per IP ( $\mathrm{N}=1,283)$.

(66\%) of IPs originating from the departments of Chemistry and Chemical Engineering consulted more than one journal, $50 \%$ more than two journals, and $37 \%$ more than three. In comparison, the numbers for Molecular Biology \& Genetics were $40 \%, 18 \%$, and $11 \%$ respectively.

\section{Total Downloads Can Estimate User Population}

There is a very strong relationship between the number of article downloads and the number of unique IP addresses $(\mathrm{Rsq}=0.92)$. Therefore, by just knowing the number of article downloads for a journal, it is possible to approximate its user population with a relative degree of accuracy.

To better understand this linear relationship, a linear regression was run with the number of article downloads as the predictor (independent variable) and the number of users as the dependent variable.

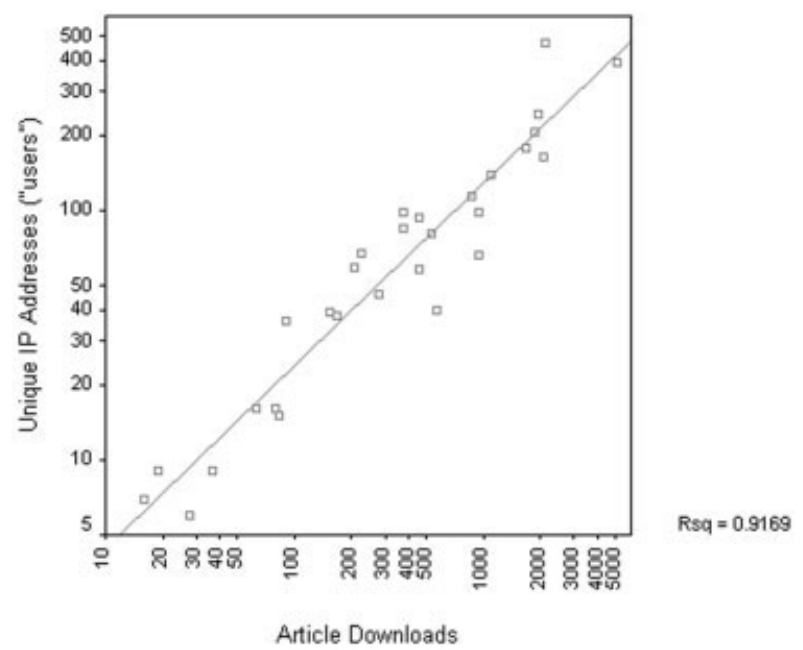

FIG. 3. Relationship between number of article downloads and number of IP addresses ("users") for each ACS journal ( $\mathrm{N}=29)$. 
The regression equation is:

$$
\mathrm{Y}_{\mathrm{users}}=\beta_{0}+\beta_{\text {downloads }} \chi
$$

where,

$$
\begin{gathered}
\beta_{0}=20.96(95 \% \text { C.I. }=8.32-33.59) \\
\beta_{\text {downloads }}=0.093(95 \% \text { C.I. }=0.078-0.107) .
\end{gathered}
$$

In this equation, $\beta_{0}$ represents the $\mathrm{Y}$-intercept and $\beta_{\text {downloads }}$ represents the slope of the line. For every additional article download, we can expect another 0.093 users. Turning this around, each user represents approximately $11 \pm 3.5$ of the total downloads. For example, if we observe a new ACS journal to have a total of 1,000 downloads, we can estimate its user population as approximately 114 individuals, and be $95 \%$ confident that it will lie somewhere between 86 and 141.

\section{Single-Title Uses}

As previously detailed, IPs associated with the departments of Chemistry and Chemical Engineering were found to be the core users of the ACS online journals, both in their numbers of downloaded articles (depth of use) and in the number of journals consulted (breadth of use). Still, there was substantial use of the ACS journals by IPs associated with other departments. An analysis of single-title uses can alert us of the degree interdisciplinary interest for ACS journals.

Fifty-five percent $(55.5 \%)$ of IPs downloaded articles from just one journal in the ACS package (Fig. 1), but only accounted for $12 \%$ of the total downloads. The same useratio is observed with each journal, with two major exceptions: Biochemistry and the Journal of Agricultural and Food Chemistry. Both of these journals experienced greater percentages of single-title users than the other journals (Fig. 4).

Of the 468 IPs downloading articles from Biochemistry, $21 \%$ of them were single-title users, accounting for $30 \%$ of the total downloads for this journal. There were no outliers in this group that could have skewed the findings. These single-title users downloaded a median of 2 articles per user, and no more than 16 downloads for the highest user. The number of users for Biochemistry has remained relatively stable for the last year and a half, although growing slowly along with other ACS journals in general, suggesting that a class reading or unique event was not contributing to the observation. The high number of single-title uses may be explained by researchers from molecular biology, genetics, and related fields using Biochemistry in exclusion of other ACS journals. Of the 67 IP addresses that were traceable to the department of Molecular Biology \& Genetics, 43 of them used Biochemistry, accounting for $30 \%$ of all article downloads from this group.

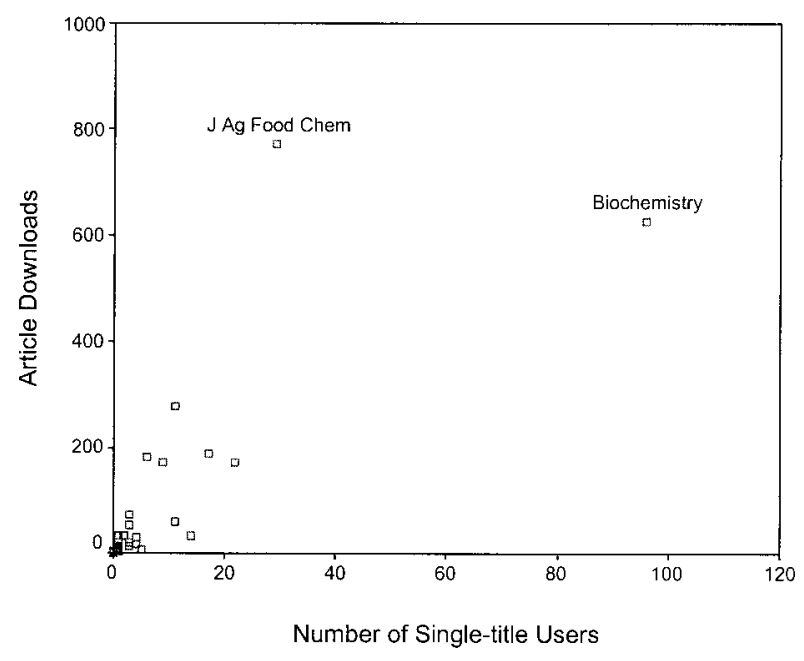

FIG. 4. Patterns of single-title journal users $(\mathrm{N}=712)$.

For the Journal of Agricultural \& Food Chemistry, single-title users accounted for only $14 \%$ of the total users, but made up $41 \%$ of the total downloads. While the median number of downloads by single-title users was only 2 , there was one individual IP that was responsible for downloading a total of 227 articles, accounting for over $12 \%$ of the use for this title. This IP was traced to a student dormitory. Even when this individual was pulled from the analysis, singletitle users still accounted for $34 \%$ of the total downloads. Like Biochemistry, the Journal of Agricultural \& Food Chemistry appears to be a core journal to food scientists-a group of researchers who are unlikely to be heavy users of other journals in the ACS package. Of the 40 IP addresses that were traceable to the Food Sciences Department, 20 of them downloaded articles from J. Agr Food Chem, accounting for $27 \%$ of all downloads from this group. Based on the JCR for 2001, J. Agr Food Chem was the highest cited journal within the field of Food Science \& Technology.

\section{Relationship Between Journals and Article Downloads}

Plotting each of the 1,283 IPs by the number of journals consulted and the number of articles downloads reveals an intriguing relationship. The relationship is not linear, but quadratic, meaning that users that rely on more titles tend to download many more articles than expected from an additive relationship (Fig. 5). A square-root transformation of the dependent variable (article downloads) straightened the regression line (Fig. 6), with no violation of the assumptions for linear regression, confirming that a square-law is in effect. All data points beyond three standard deviations of the regression line were considered outliers and were further investigated.

Most of the outliers were heavy users of two or more journals, one of which was the Journal of the American Chemical Society. Most came from the Chemistry Department with a few others coming from departments with related subject fields (i.e., Food Science, Engineering, Phys- 


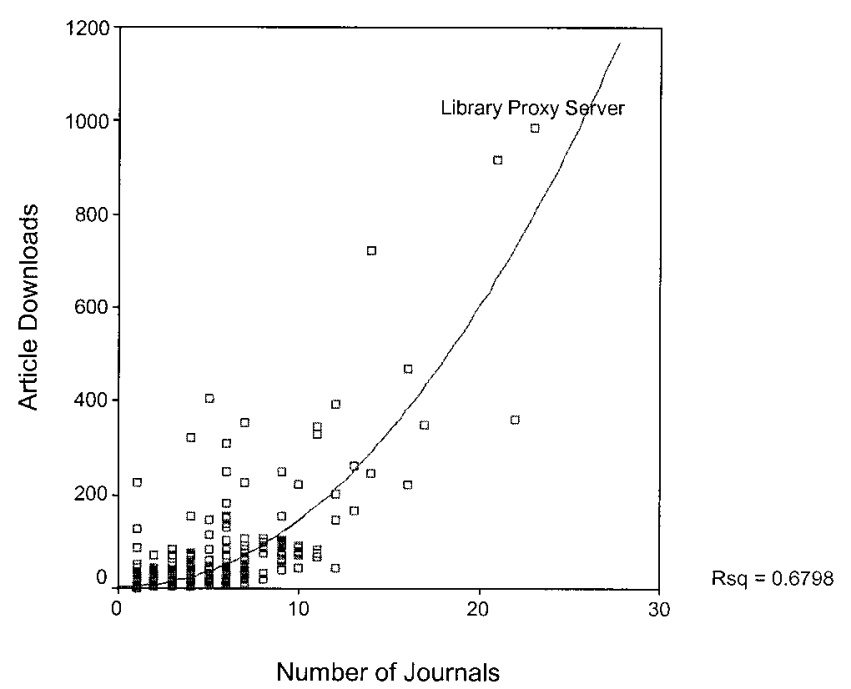

FIG. 5. Quadratic relationship between journals consulted and articles downloaded $(\mathrm{N}=1,283)$.

ics). None of the outliers represented computers located in libraries. These data suggest that the function of JACS as a multi-disciplinary browsing journal for chemists appears to hold online as it does in print.

The IP that accounted for the largest number of downloaded articles over the largest number of journals was the Library Proxy Server. The fact that this data-point does not radically deviate from the rest of the model suggests that the proxy server can be regarded as a sample of the rest of the user population. A one-sample t-test comparing journal use of the proxy server with the entire population confirms this observation significantly $(P<0.001)$.

The behavior associated with library computers also does not appear to deviate from the rest of the user-population. Individuals using these computers downloaded a median of 4 articles from only 1 journal-identical to the rest of the population. Unfortunately this subpopulation was too small to analyze if there was any difference in the statistical distribution of the data with the rest of the user population.

\section{Discussion}

To date, most online publishers only provide aggregated statistics for individual journal titles, summarized monthly, quarterly, or annually. Without understanding the variation in user behavior, it is difficult to understand what these aggregate statistics represent.

This study illustrates that for ACS titles, the vast majority of users download few articles and consult few journals. It also illustrates that a small number of heavy users can have an extremely large effect on the number of total downloads. And lastly, the relationship between journals consulted and article downloads is quadratic. While the raw use statistics may not be comparable to other ACS subscribing institutions, it is believed that the usage patterns identified may be generalizable.
Despite the existence of heavy users, this study suggests that the size of the user population can be estimated by just knowing the total number of downloads per title. Each "user" (IP) can be represented by approximately $11 \pm 3.5$ downloads. This prediction method may be useful for estimating user populations of other publishers' journals when only total number of downloads are provided.

\section{Relationship of Journal Versus Article Downloads}

The quadratic relationship between the number of journals consulted and number of articles downloaded per user suggests that some power law (specifically an inverse square law) may be in effect (Figs. 5 and 6).

In studying the frequency distribution of scientific productivity, Alfred Lotka counted the number of times authors were indexed in Chemical Abstracts and Auerbach's Tables. He discovered that the distribution followed an inverse square law (known as Lotka's Law), whereby the number of authors contributing $n$ articles, follows $1 / n^{2}$. The pattern of journals consulted per user in this study (Fig. 2) shows a better fit to Lotka's distribution than to the Poisson, exponential, or normal distributions.

The distribution of the number of downloads per user makes a rather poor fit for Lotka's distribution (Fig. 1). The deviation of the pattern of article use from the pattern of journal use may have to do with possible interdependence of observed events and the definition of "user."

Most statistical analyses assume that observed events are independent from each other. One download is assumed to be completely independent from any other download. Based on experience alone, we know this to be untrue. Users browsing for articles regularly download more than one article per visit, and may even download the same article again in different formats (i.e. HTML then PDF), multiple times per session, or at different times during the threemonth window. We also made several assumptions about

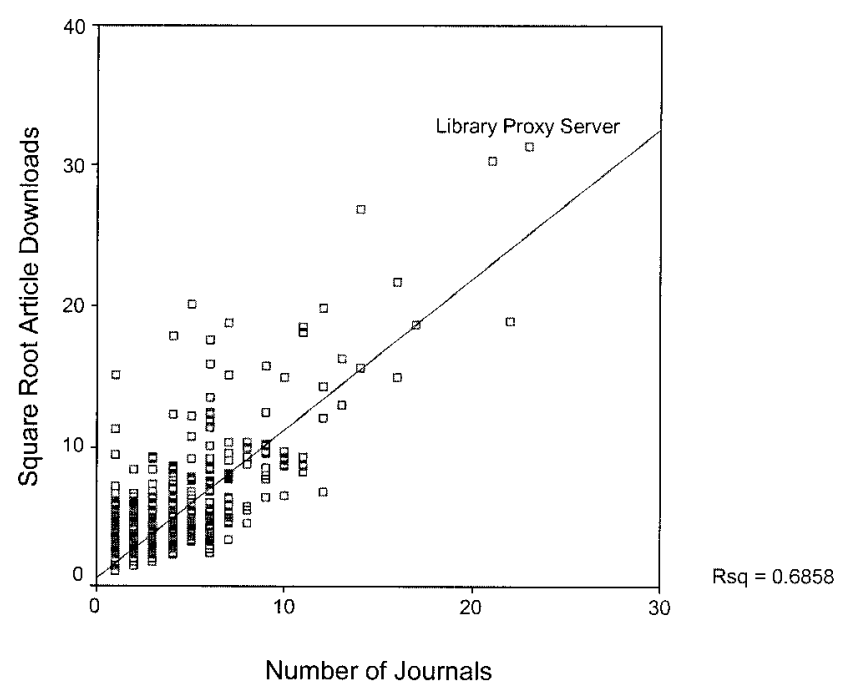

FIG. 6. Square root transformation yields linear relationship $(\mathrm{N}=1,283)$. 
the "user." Each IP address was equated with a single user, with the full understanding that some of the IPs represent aggregated users, such as computers in departmental labs, public computing clusters and libraries.

In addition, the users of the ACS journals consist of multiple subpopulations (chemists, physicists, molecular biologists, food technologists, etc.), who have different literature-usage patterns as detailed above in our Literature Review. IPs associated with the departments of Chemistry and Chemical Engineering were found to download more articles and consult more journals than any other subpopulation. Still, other departments showed substantial use of these journals but were limited to a smaller number of titles and article downloads.

\section{User Intent}

Interviews and questionnaires following the SuperJournal project indicated that users preferred reading HTML from the screen, but PDF format when they print. Ken Eason reported that only a small minority of users would read articles from the screen (Eason et al., 2000). In this study, HTML downloads accounted for less than $10 \%$ of use for all journals. Chemists are known to be heavy browsers of the literature, so why are they not using the HTML feature for browsing? It may be possible that the majority of downloads were for known articles. The existence of many heavy-users of single titles seems to suggest that some of our users are heavy browsers, but further analysis indicates that these heavy users also show a preference for PDF over HTML. Several of the ACS online journals offer tables of contents with graphics, which may be assisting users to browse online without downloading articles. With only the evidence from HTML versus PDF downloads, it appears from these data that users of ACS online journals are using the system primarily as a networked photocopier, for the purposes of creating print-on-demand copies of articles rather than for browsing and knowledge discovery.

While we can document the use of ACS online journals, it is still not known how Cornell patrons are discovering individual articles. Are they linking to an article from within the ACS system, from a bibliographic database like Chemical Abstracts, or directly from a citation in another article? Much more quantitative and qualitative research is desperately needed to better understand individual online behavior. In its absence, any quantitative evaluation can be easily misinterpreted.

\section{Acknowledgments}

Great appreciation goes to Greg Lawrence at Cornell University, for helping in the preparation of the data; Tina
E. Chrzastowski, University of Illinois at Urbana-Champaign, and Stephen J Bensman, Louisiana State University for their assistance in the explanation of the results; and Chuck Hamaker, University of University of North Carolina at Charlotte for his review of the manuscript.

\section{References}

American Chemical Society. (2002). Journals \& Magazines [online]. Retrieved Dec 15, 2002, from http://pubs.acs.org/about.html

Blecic, D.D., Fiscella, J.B., \& Wiberly Jr., S.E. (2001). The measurement of use of Web-based information resources: An early look at vendorsupplied data. College \& Research Libraries, 62, 434-453.

Brown, C.H. (1956). Scientific serials: Characteristics and lists of most cited publications in mathematics, chemistry, geology, physiology, botany, zoology, and entomology (Vol. ACRL Monograph no. 16). Chicago: Association of College and Reference Libraries.

Brown, C.M. (1999). Information seeking behavior of scientists in the electronic information age: Astronomers, chemists, mathematicians, and physicists. Journal of the American Society for Information Science, 50, 929-943.

COUNTER (2003). COUNTER: Counting Online Usage of Networked Electronic Resources [Web]. Retrieved March 7, 2003, from http:// www.projectcounter.org/index.html

Davis, P.M. (2002). Patterns in electronic journal usage: Challenging the composition of geographic consortia. College \& Research Libraries, 63, 484-497.

Eason, K., Richardson, S., \& Yu, L. (2000). Patterns of use of electronic journals. Journal of Documentation, 56, 477-504.

Entlich, R., Garson, L., Lesk, M., Normore, L., Olsen, J., \& Weibel, S. (1995). Making a Digital Library: The Chemistry Online Retrieval Experiment. Communications of the ACM, 38, 54.

ISI. (2001). Journal Citation Reports. Retrieved Dec, 2002, from http:// www.isinet.com/isi/products/citation/jcr/

King, D., \& Tenopir, C. (1999). Using and reading scholarly literature. Annual Review of Information Science and Technology, 34, 423-477.

Lotka, A.J. (1926). The frequency distribution of scientific productivity. Journal of the Washington Academy of Sciences, 16, 317-323.

Nicholas, D., Huntington, P., \& Watkinson, A. (2002, October). Digital journals, big deals and online searching behaviour: A pilot study. Paper presented at The Consortium Site License-Is it a sustainable model?, London, UK

Olsen, J. (1994). Electronic journal literature: Implications for scholars. Westport: Mecklermedia.

Pullinger, D.J. (1994). The SuperJournal Project. Philadelphia: Institute of Physics Publishing.

Sanville, T.J. (2001). A method out of the madness: OhioLINK's collaborative response to the serials crisis three years later: A progress report. The Serials Librarian, 40, 129-155.

Stewart, L. (1996). User Acceptance of Electronic Journals: Interviews with Chemists at Cornell University. College \& Research Libraries, 57, 339-349.

Tenopir, C., \& King, D. (2000). Toward electronic journals: Realities for scientists, librarians, and publishers. Washington: Special Libraries Association.

Tenopir, C., \& King, D. (2002). Reading behaviour and electronic journals. Learned Publishing, 15, 259-265.

Tenopir, C., \& Read, E. (2000). Patterns of database use in academic libraries. College \& Research Libraries, 61, 234-246. 